\title{
On the Classification of Microsoft-Windows ransomware using hardware profile
}

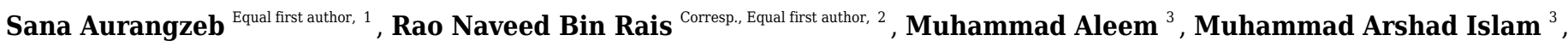 \\ Muhammad Azhar Iqbal ${ }^{4}$ \\ 1 Department of Computer Science, National University of Modern Languages, Islamabad, Islamabad, ICT, Pakistan \\ 2 College of Engineering and Information Technology, Ajman University, Ajman, Ajman, United Arab Emirates \\ 3 Department of Computer Science, National University of Computer and Emerging Sciences, Islamabad, Islamabad, ICT, Pakistan \\ 4 School of Information Science and Technology (SIST), Southwest Jiaotong University, Chengdu, China \\ Corresponding Author: Rao Naveed Bin Rais \\ Email address: r.rais@ajman.ac.ae
}

Due to the expeditious inclination of online services usage, the incidents of ransomware proliferation being reported are on the rise. Ransomware is a more hazardous threat than other malware as the victim of ransomware cannot regain access to the hijacked device until some form of compensation is paid. In the literature, several dynamic analysis techniques have been employed for the detection of malware including ransomware; however, to the best of our knowledge, hardware execution profile for ransomware analysis has not been investigated for this purpose, as of today. In this study, we show that the true execution picture obtained via a hardware execution profile is beneficial to identify the obfuscated ransomware too. We evaluate the features obtained from hardware performance counters to classify malicious applications into ransomware and nonransomware categories using several machine learning algorithms such as Random Forest, Decision Tree, Gradient Boosting, and Extreme Gradient Boosting. The employed data set comprises 80 ransomware and 80 non-ransomware applications, which are collected using the VirusShare platform. The results revealed that extracted hardware features play a substantial part in the identification and detection of ransomware with F-measure score of 0.97 achieved by Random Forest and Extreme Gradient Boosting. 


\section{On the Classification of Microsoft-Windows}

3 Ransomware Using Hardware Profile

4

5

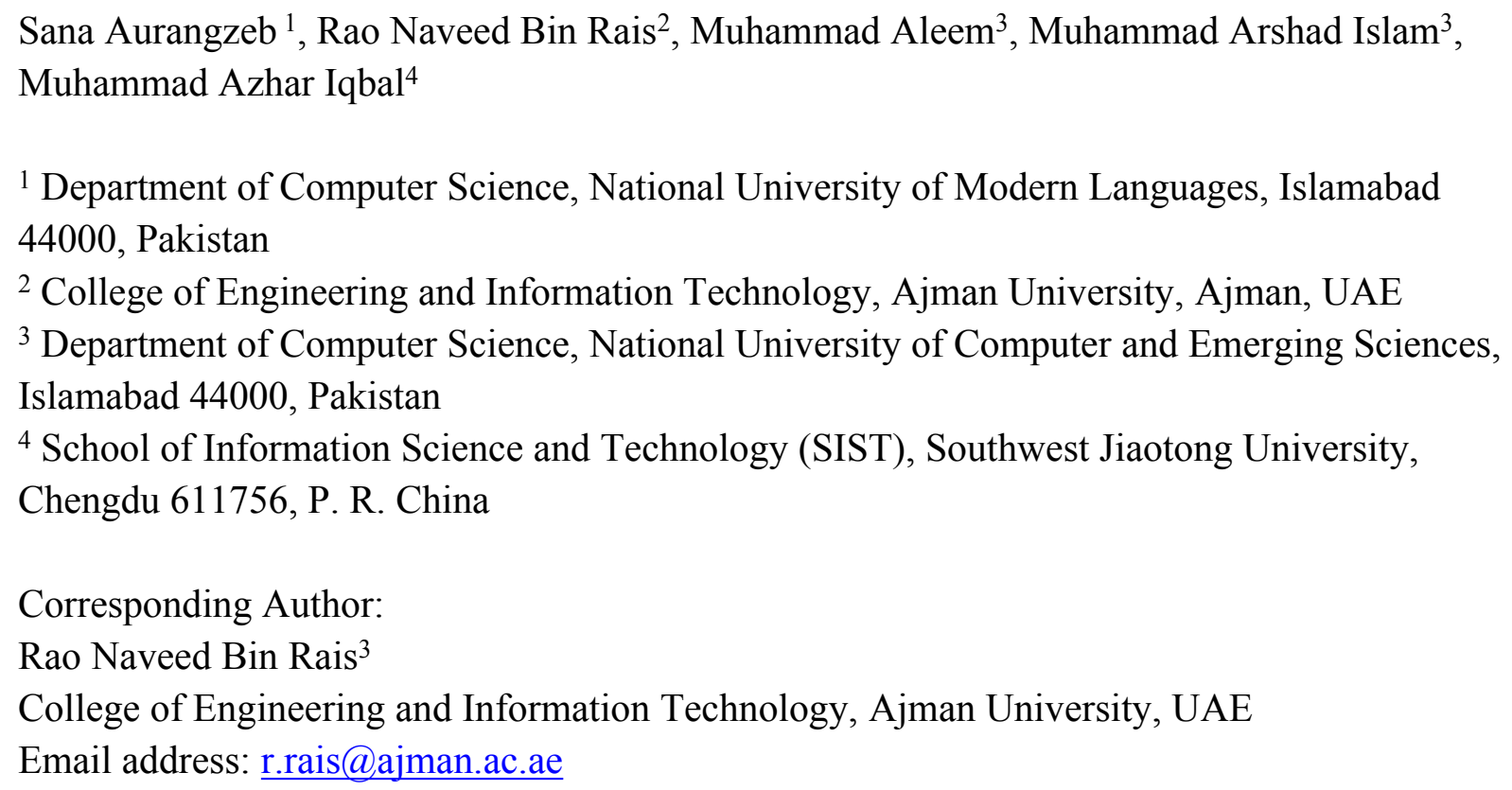

\section{Abstract}

Due to the expeditious inclination of online services usage, the incidents of ransomware proliferation being reported are on the rise. Ransomware is a more hazardous threat than other malware as the victim of ransomware cannot regain access to the hijacked device until some form of compensation is paid. In the literature, several dynamic analysis techniques have been employed for the detection of malware including ransomware; however, to the best of our knowledge, hardware execution profile for ransomware analysis has not been investigated for this purpose, as of today. In this study, we show that the true execution picture obtained via a hardware execution profile is beneficial to identify the obfuscated ransomware too. We evaluate the features obtained from hardware performance counters to classify malicious applications into ransomware and non-ransomware categories using several machine learning algorithms such as Random Forest, Decision Tree, Gradient Boosting, and Extreme Gradient Boosting. The employed data set comprises 80 ransomware and 80 non-ransomware applications, which are collected using the VirusShare platform. The results revealed that extracted hardware features play a substantial part in the identification and detection of ransomware with F-measure score of 0.97 achieved by Random Forest and Extreme Gradient Boosting. 
40 Keywords: malware; ransomware; performance counters; classification; machine learning

41

\section{Introduction}

43 Over the past several years, an exponential increase has been reported in ransomware attacks. Ransomware is the sub-class of malware that hijacks a device and blocks the victim to access the data until a compensation of some form is made. Typically, this compensation is in the form of money to concede access back to the victim. Ransomware has the ability to harmfully affect various kinds of devices such as personal computers, servers, smartphones, tablets, etc. For instance, multiple new variants of ransomware including WannaCry, JAFF, Petya have been reported in 2017 (Hampton, et al., 2018). On May 12, 2017, within the span of a few hours, the WannaCry ransomware (Maurya, et al., 2018) infected more than 70,000 desktop devices in over 150 countries across the globe (Grant \& Parkinson, 2018).

The financial loss incurred due to ransomware can be quite devastating. For instance, CryptoWall_v3 ransomware (Cyber Threat Alliance, 2016; Sgandurra, et al., 2016) caused the loss of an estimated \$325 million in the US from November 2015 to June 2016.). In 2018, it was reported that $45 \%$ of the companies paid a ransom to recover the data stored on infected machines, which increased to 58\% in the year 2019 (Ramesh and Menen 2019). Another ransomware attack, triggered by CryptoWall_v4 ransomware resulted in a loss of \$7.1 million worldwide (Cyber Threat Alliance, 2016). Recently reported ransomware attacks involving NotPetya and WannaCry are estimated to inflict the costs around \$18 billion (Davies, et al. 2020). These attacks wreaked havoc in systems of various world organizations by halting and damaging their daily operations. It seems that losses caused by ransomware will probably exceed $\$ 20$ billion by the end of the year 2021 (shown in Figure 1) as reported by the Global ransomware-protection report (Ramesh and Menen, 2020; Chung, 2019).

Although, malware is deemed a great threat over the years, yet ransomware is an even more daunting threat compared to other malware due to its attacking and demanding nature (i.e., expecting a ransom in return). The classification of ransomware from traditional malware is essential because of their higher damaging impact in terms of informative data and financial loss. Compared to typical malware, it is challenging to identify and kill ransomware even when it is discovered, and the damage can be potentially irreparable even after its deletion (Al-remy, et al., 2018) and (Zhang, et al., 2019). Hence, we require proactive and aggressive techniques to handle ransomware. Moreover, it is very challenging to recognize and isolate the malware from ransomware due to the similarity in nature. A ransomware is more menacing than malware, as it not only damages the system and results in loss of control from the system but also demands compensation in return. Therefore, there is a need to have the proper distinction of ransomware from other malware (Aurangzeb et al, 2017; Kok et al., 2019 and Zhang, et al., 2019) to save billions of dollars in financial losses (Davies, et al 2020). 
80 Before analyzing the ransomware, one of the mandatory steps is the accurate identification of a

81

82

83

84

85

86

87

88

89

90

91

92

93

94

95

96

97

98

99

100

101

102

103

104

105

106

107

108

109

110

111

112

113

114

115

116

117

118

119

particular type of ransomware and differentiating it from other typical malware. Broadly, malware analysis techniques are categorized as 1) static and 2) dynamic analysis (Chen, et al., 2017). Besides, various researchers have employed the combinations of static and dynamic techniques in the form of hybrid analysis techniques. The procedure of scrutinizing a potential malware without executing the program is referred to as static analysis, whereas, the analysis performed via observing the execution behavior of a malware is known as dynamic analysis. Most contemporary state-of-the-art dynamic analysis techniques detect and classify ransomware that hides itself using various obfuscation techniques such as packed programs, compressed, or data transformation, indirect addressing, etc. (Behera \& Bhaskari, 2015). A ransomware employs different hijacking strategies such as behaving like an adware resulting in unwanted advertisements or hiding itself using rootkits to bypass Anti-Viruses (AV) (Demme, et al. 2013). A rootkit is a malware that alters the operating system (OS) and resides in the system for a prolonged period (Aurangzeb, et al., 2017). Today, various anti-viruses tackle malware to dampen their caused and expected damages. However, the techniques employed by the antiviruses are often limited to the prior knowledge (e.g., signatures, etc.) and there is a need to have more comprehensive dynamic analysis that could detect ransomware, employing the obfuscation techniques (Demme, et al., 2013), utilizing hardware performance counters.

Hardware Performance Counters (HPCs) have been typically used by the programmers to analyze and measure the performance of applications and to identify the execution bottlenecks of a program (Beneventi, et al. 2017; Kuruvila et al., 2020). Initially, HPCs have been employed for investigating the static and dynamic analysis of programs to detect any malicious amendments as mentioned in (Alam, et al.,2020) and (Malone, et al., 2011). Several studies (Das et al., 2019; Demme, et al., 2013; Singh, et al., 2017; and Wang, et al., 2016) discuss potential implications of using Hardware Performance Counters (HPC) for application analysis, and the majority of them suggest that hardware execution profile can effectuate the detection of malware (Demme, et al., 2013; Singh, et al., 2017; and Wang, et al., 2016; Kuruvila et al., 2020). Another study (Xu, et al., 2017) has utilized the hardware execution profiles to detect malware using machine learning algorithms, as malware changes data structures and control flow, leaving fingerprints on accesses to program memory. In this respect, they proposed a framework for detecting malware from benign applications that uses machine learning to classify malicious behavior of malware based on access patterns of virtual memory. (Zhou, et al., 2018) investigated whether HPCs are useful in differentiating the malware from benign applications. However, the study did not consider malware as ransomware. However, utilizing the hardware performance measurements and the profile of the low-level execution behavior has not been previously studied for the analysis and detection of ransomware applications. We argue that ransomware reveals itself by exhibiting peculiar patterns in HPCs (e.g., through clock cycles, cache misses and hits, branch instructions and misses, retired instructions, etc.).

Peer) Comput. Sci. reviewing PDF | (CS-2020:09:52627:1:1:NEW 12 Dec 2020) 
120 In this paper, we present a framework based on dynamic analysis that mainly focuses on the 121 classification of ransomware from non-ransomware. This paper contemplates HPCs to detect

122 Microsoft Windows-based ransomware by analyzing the execution behavior of ransomware. We 123 primarily focus to determine the potential use of HPCs in analyzing and proactively detecting 124 ransomware. Moreover, the classification of ransomware from malware is imperative because 125 the damages caused by ransomware drastically ensure the data and monetary loss. To address 126 this concern, we propose a mechanism that utilizes the application execution profile for the 127 classification and detection of ransomware from non-ransomware. For classification, the 128 application's hardware related performance features are extracted from the data set of 160 129 malware (consisting of 80 ransomware and 80 non-ransomware). Afterward, these features are 130 fed to some well-known machine learning classification models such as Decision Tree (Kohavi, 131 1996), Random Forest (Liaw, et al., 2002), Gradient Boosting (Friedman, 1999), and Extreme 132 Gradient Boosting (XGBoost) (Chen, et al., 2015). These four classifiers are generally used for 133 classification tasks of various applications including spam detection, face recognition, and 134 financial predictions (Jordan and Mitchell, 2015; Kuruvila et al., 2020), etc. We employ these 135 four classifiers as part of the proposed methodology to analyze their performance for 136 ransomware detection. These models perform binary classification of malicious software into 137 ransomware or non-ransomware classes. In summary, the main contributions of this paper are as 138 follows:

139

140

- In-depth analysis of the current state-of-the-art to identify the merits and demerits of several existing approaches;

- A novel mechanism for the classification and detection of malicious applications into ransomware and non-ransomware; and

- An empirical investigation of the HPCs against state-of-the-art dynamic techniques using machine learning classifiers;

The outcomes revealed that both the random forest and extreme gradient boosting classifier has outperformed decision tree and gradient boosting by attaining accuracy of 0.97 for classification.

The rest of the paper is organized as follows. Section 2 describes the related work. Section 3 presents the proposed methodology, dataset, and feature extraction mechanism. In Section 4, the experimental setup details, results, and related discussions are presented and Section 5 concludes the paper.

154 155

\section{Related Work}


156 For dynamic analysis, it is necessary to collect key ransomware features at runtime. Most of the 157 dynamic analysis-based research studies exploit the renowned malware databases ${ }^{1}$ for the 158 acquisition of malicious software and use quarantine environments (such as Cuckoo's sandbox 159 (Kaur, Dhif and Singh 2017)) to execute the applications.

160

161

162

163

164

165

166

167

168

169

170

171

172

173

174

175

176

177

178

179

180

181

182

183

184

185

186

187

188

189

190

191

192

In (Zavarsky and Lindskog 2016), the authors presented an experimental analysis of Microsoft Windows and Android-based ransomware. This analysis demonstrates that ransomware detection could be performed by monitoring the abnormalities in the file system and registry activities. It is shown that a significant number of ransomware families exhibit very similar characteristics. Moreover, the authors concluded that changes in a particular set of registry keys are important aspects to be analyzed for ransomware detection. The authors discovered that Microsoft Windows 10 is reasonably effective against ransomware attacks. Moreover, this study also revealed that for the Android platform, the Android Manifest file and the permissions (required by an app) should also be considered for ransomware detection. There is a lot of work (Alzahrani and Alghazzawi, 2019; Victoriano 2019) related to Android malware detection using machine learning approaches to classify malware families. Authors in (Scalas et al., 2019) focus on ransomware classification and proposed a learning-based detection strategy. The proposed scheme relies on system's API information such as packages, classes, and methods related traces. The proposed scheme is capable to differentiate and classify generic malware, ransomware, and goodware. The experimental results highlight the significance and effectiveness of using system API information for Android ransomware classification.

Several researchers utilized the hash information (i.e., comparing hash values) to detect the CryptoLocker ransomware (Song, Kim, and Lee 2016). The affected systems are recovered by the following ways: 1) process CryptoLocker, 2) comparing hash information with the encrypted data files 3 ) validating the key using the key-index information stored therein, and 4) proceeding to decode. Generally, this type of process consumes a lot of time for ransomware detection with a potential risk that another ransomware appears until a security company comes up with decryption keys of the old ransomware. Moreover, additional analysis is needed to detect new patterns of ransomware as the hackers persistently come up with new variants of ransomware. On the Android platform, another technique is proposed (Song, Kim, and Lee 2016) to prevent ransomware intrusion. The technique requires intense monitoring of the execution processes and analysis of the particular file directories using the statistical techniques, such as Next-generation Intrusion Detection Expert System (NIDES) (Anderson et al, 1995) using the processor, memory usage, and $\mathrm{I} / \mathrm{O}$ rates, to uncover the applications exhibiting abnormal behavior (Song, Kim and Lee 2016).

\footnotetext{
${ }^{1}$ www.virusshare.com
} 


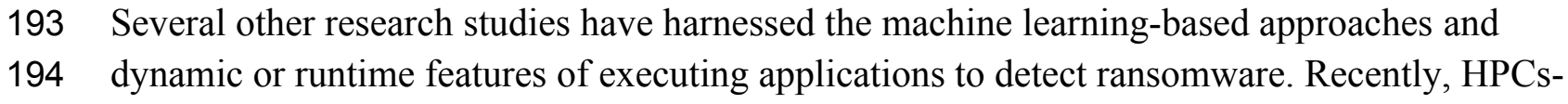

194

195

196

197

198

199

200

201

202

203

204

205

206

207

208

209

210

211

212

213

214

215

216

217

218

219

220

221

222

223

224

225

226

227

228

229

230

231

232 based events and their features are being used widely in research to detect side-channel attacks and ransomware (Or-Meir et al., 2019). (Alam et al. 2020) have used HPCs features to detect malware from benign applications. The authors proposed an anomaly detection technique to identify malicious ransomware in a few seconds with very few false positives using Recurrent Neural networks (RNN). However, only five hardware performance measures i.e., instruction, cache-references, cache-misses, branches, and branch-misses are investigated, whereas the authors investigation is with one type of ransomware only, which was WannaCry. In (Kadiyala et al.,2020), only four hardware performance aspects were considered. Maiorca et al., (Maiorca et al. 2017) proposed a supervised machine learning-based procedure, R-PackDroid, to detect Android ransomware, which is a light-weight technique and does not require prior knowledge of ransomware's encryption mechanisms. However, the R-PackDroid technique uses fully encrypted code-files and is unable to analyze the applications that load the code at run-time. The R-PackDroid can be incorporated with the other dynamic analysis methods, such as the approach proposed by (Kimberly et al., 2015). Moreover, R-PackDroid based application analysis strongly depends on the parsing capabilities of the ApkTool framework.

(Narudin, et al. 2016) has presented a machine learning-based malware analysis approach based on the anomaly detection mechanism. The results indicated that Bayes network and Random Forest classifiers produce accurate results by attaining $99.97 \%$ True-Positive Rate (TPR) as compared to the multi-layer perceptron technique with only $93.03 \%$ TPR using the MalGenome data set. However, the accuracy of this scheme dropped to $85 \%$ for the latest malware experiments.

Desktop ransomware can easily bypass any counter-measures and thus results in the seizure of personal data. Authors (Al-rimy et al., 2018) presented an effective mechanism for early diagnosis and avoidance of the crypto-ransomware, which is based on machine learning techniques (One-Class SVM and n-gram technique (Zhang, et al., 2015)) and comprises three modules: 1) pre-processing, 2) features engineering and 3) detection module. The authors employed an adaptive anomaly detection mechanism that handles the dynamic characteristics of systems and frequently updates the normal profile built from the feature extraction (Al-rimy, et al., 2017) to improve the accuracy of detection.

The study (Kharraz, Roberstson, et al. 2015) has presented the analysis of ransomware families (the year 2006-2014) and concludes that the suspicious activity of file systems should be observed for ransomware detection. For instance, the changes in the types of I/O Request Packets (IRP) or the Master File Table (MFT) are usually formed to access the file system. A considerable number of ransomware families share related features as a core part of the attacks; however, there still lacks a reliable destructive function to successfully infect files of victims. In

Peer] Comput. Sci. reviewing PDF | (CS-2020:09:52627:1:1:NEW 12 Dec 2020) 
233 Table 1, we recapitulate several other prominent ransomware detections (Yang, et al. 2015;

234 Andronio, Zanero and Maggi 2015; Kharraz, Arshad, et al. 2016) and prevention (Ahmadian, 235 Shahriari and Ghaffarian 2015; Kim, Soh and Kim 2015; Lee, Moon and Park 2016; Brewer 236 2016) techniques. Recently, deep neural networks and convolutional neural networks (CNNs) 237 have shown remarkable performance in the area of object recognition (Simonyan and Zisserman 238 2014). The deep convolutional neural networks can outperform the other approaches like Natural 239 Language Processing (NLP), if the training is performed using large datasets (Liu and Liu 2014; 240 Zhang et al., 2015). Due to the limited dataset, we have employed supervised machine learning 241 algorithms. Moreover, the core objective of our proposed scheme is to gauge the effectiveness of 242 the hardware execution profile (i.e., a truly dynamic environment) for the classification of 243 244 ransomware/non-ransomware.

Besides, the performance counters exhibit the true application execution behavior and are being employed by the researchers to analyze application performance (Mucci, et al., 1999) (Bahador, et al., 2014) (Demme, et al., 2013). In (Basu et al., 2019) authors have used hardware performance counters to detect Android malware, and in another similar work (Bahador et al., 2019) authors have presented a heuristic (using signature-based features and hardware performance counters) to detect and classify malware. Their approach is only suitable for malware detection that are invoked as standalone applications and are not dependent on other host applications. In summary, none of the existing dynamic analysis techniques utilizes the important dynamic feature such as HPCs to detect Windows platform based malicious applications. Although, there are few approaches available that classify a benign application from ransomware, however, to the best of our knowledge no other approach (utilizing hardware performance counters) classified malware into the subclass of ransomware/non-ransomware on the Windows platform. Malware can employ obfuscation techniques to deceive static analysis based anti-viruses. Furthermore, runtime behavior cannot be obfuscated and can be detected using dynamic analysis. We believe this aspect should essentially be exploited and the hardware

260

261

262

263

264

265

266

267

268

269

270

271 execution profile should be utilized to execute applications for ransomware detection. Based on these facts, we argue that HPCs are useful features that could be utilized for the detection and classification of ransomware. In this study, we employ various machine learning classifiers such as Decision Tree, Random Forest, Gradient Boosting, and Extreme Gradient Boosting along with the HPCs to address the following questions:

(1) How different are ransomware from malware at runtime considering machine learning techniques?

(2) Which of the hardware performance counters (HPCs) play a vital role in ransomware detection?

\section{Motivation and Methodology}


272 The dynamic analysis holds adequate potential to accurately detect the threat of ransomware

273 because an executable program cannot hide its true characteristic. Therefore, most of the anti-

274 virus vendors rely on automated dynamic analysis mechanisms to detect new variants of

275 ransomware. Most of the antiviruses apply the heuristics combined with the behavior analysis to

276 deduce whether an executable is benign or malware (Sgandurra et al. 2016).

277

278

279

A wide range of HPCs i.e., clock cycles, cache hits, cache misses, branch instructions, branch

280 misses, retired instructions, etc. are used to observe the behavior of an executing application

281

282

283

284

285

286

287

288

289

290

291

292

293

294

295

296

297

298

299

300

301

302

303

304

305

306

307

308

309

310

311 (Chiappetta, Savas and Yilmaz 2016). Usually, the symmetric encryption marks the cache-based events while the asymmetric encryptions do have an impact on the instruction and branching events as explained in (Alam, et al., 2020). HPCs have been harnessed by many application developers to identify the computation and memory bottlenecks to improve the performance and reliability of the executing applications (Chiappetta et al. 2016). In this study, we utilize 11 performance counters for the classification of ransomware. For classification, we train the employed machine learning classifiers to analyze the dynamic behavior of ransomware and nonransomware malicious programs. Moreover, the classification of Ransomware from Traditional Malware is essential due to the intensity of the damage caused in terms of financial loss. Unlike traditional malware, it is more troublesome to identify and kill ransomware even when it is discovered, and the damage is irreparable even after its removal (Al-remy, et al., 2018) and (Zhang, et al., 2019). Hence, it is very important to recognize and isolate the malware from ransomware due to the similarity in nature. Therefore, it is required to devise a formal classification mechanism to discriminate ransomware from other non-ransomware (Zhang, et al., 2019), (Aurangzeb et al, 2017), and (Kok et al., 2019) to avoid billions of transactions in the name of ransom.

\section{DATASET COLLECTION}

For the experimentation, we have obtained randomly selected 160 Windows-based malware from VirusShare. VirusSharerepository provides the dataset related to ransomware and many other types of malicious applications of the Windows platform (in addition to the other platforms such as Android, Linux, etc.). It is frequently updated and at presently contains the latest malicious applications contributed by the community (Kouliaridis and Kambourakis 2020). Due to the diversity, the VirusShare platform is very popular in the research community. We collected the dataset from VirusShare related to Windows-based malicious applications. After static analysis of the downloaded applications, obfuscated applications are eliminated. Afterward, each malware is labeled as a non-ransomware or ransomware based on the analysis data provided by many renowned anti-viruses available via VirusShare. These labels are further validated with the tags available from VirusShare for the sake of confirmation. In this study, benign binary files are not considered because the main aim of the study is to classify between ransomware and other malicious applications. Therefore, we consider the malicious applications category Trojan (as a non-ransomware sample) due to their similarity in activities with the ransomware (Gazet 2010). 
312 The employed classifiers are trained using the behavioral features for ransomware and non313 ransomware with explicit labeling (i.e., Ransomware/Non-Ransomware). Furthermore, a disjoint 314 data set is used for training and testing purposes.

\section{FEATURE EXTRACTION}

316 All malware in the data set are executed in a quarantine environment and their data related to

317 hardware performance counters are collected using perf (an instrumentation and performance 318 analysis tool (Weaver, 2013) (Alam et al., 2020)). To ensure the reliability and accuracy of the 319 results, the mean values of three rounds of experiments are reported. We executed each application 3 times in a virtual machine (i.e., VMWorkstation 12 Pro 12.1.1 build 3770994) for

321

322

323

324 325

326

327

328

329

330

331

332

333

334

335

336

337

338

339

340

341

342

343

344

345

346

347

348

349

350 no longer than 240 seconds with different input parameters to emulate a real interactive environment. After the execution of each malicious application, the virtual machine is reset to its original state using the snapshot feature (to ensure the performance counter trace collected during the previous execution do not intermingle with the current execution).

For binary classification problem discussed above, we employ hardware performance counters as features, i.e., 1) task clock, 2) context switching, 3) CPU utilized, 4) CPU migrations, 5) page faults, 6) CPU cycles, 7) cache-misses, 8) instructions retired, 9) branches taken, 10) branchmisses, and 11) execution time, (illustrated in Table 2) to train the machine learning classifier. We have executed ransomware applications on a PC within a virtual machine and recorded the features (i.e., hardware performance counters, etc.) using perf. The perf library provides the hardware performance counters related values representing the involvement of the several important hardware features of the processor during execution. Feature selection plays a significant role in achieving precise training of the employed machine learning models; thereby attaining accurate results with efficient performance and low overhead (Li, et al., 2017). A correlation matrix among the employed features is generated to analyze the pattern that leads to the selection of features. Two features are considered negatively correlated if a change of one feature inversely impacts the value of the other feature. The features correlation analysis is presented in Figure 2. If two numerical features are highly correlated, then one of them can be ignored. Therefore, we employed a sub-set of those features which are not co-related to reduce the computation overhead during the training process of the machine learning models. Figure 2 shows that the Cache Misses related hardware feature have a low positive correlation with all the other features. On the other hand, the Task Clock feature has a strong relationship with the Context Switches, Cycles, Instructions, Branches, and Branches Misses. The features having higher rank are deemed as potential features for classification than low ranked features as shown in Table 3.

In the training phase, hardware features are extracted by executing known malware and nonmalware application in containing environment system units as shown in Figures 3a and 3b. 80\% of the employed data set is used for training and $20 \%$ is used for testing. We have used K-fold 
$351 \quad(\mathrm{k}=10)$ cross-validation mechanism and compare the ransomware detection accuracy of different 352 classifiers to make sure that the dataset is used uniformly without any biasness. This results in

353 un-biased training and testing cycles producing the results on which we could conclude with 354 confidence. For each cycle of the training/testing, a $20 \%$ testing and $80 \%$ training partition was

355 employed. The goal of supervised machine learning techniques is to find a function that is 356 trained using the employed features such that the error is minimum for the new or unseen data. In 357 the training phase, the classification model is trained using the HPCs as shown in Table 2. The 358 testing or validation methodology is performed after the training of the classifiers.

359

360 CLASSIFICATION MODEL

361

362

363

364

365

366

367

368

369

370

371

372

373

374

375

376

377

378

379

380

381

382

383

384

385

386

387

388

389

390

The machine learning classification algorithms namely Decision Tree, Random Forest, Gradient Boosting, and Extreme Gradient Boosting are used for classification purposes such as phishing detection, facial recognition, and financial predictions (Jordan and Mitchell, 2015), etc. We employ these four classifiers as part of the proposed methodology to analyze their performance for ransomware detection.

The decision tree is a tree-based classifier, which contains a root, internal nodes, and leaf nodes. The class label is assigned to each leaf node and the decisions are rendered by the internal nodes (Tan, et al., 2006). Random Forest (RF) classifier is based on a combination of multiple decision tree predictors such that each tree depends on the values of a random vector sampled independently and with the same distribution for all trees in the forest (Xuan, et al. 2018). The Extreme Gradient Boosting XGBoost and Gradient Boosting follow the same basic principle however, there are a few differences in their modeling details. Specifically, extreme gradient boosting utilizes a more regularized model formalization to control the over-fitting problem that may occur due to linear fitting over noisy data to provide better performance (Jbabdi, et al., 2012). For the Decision Tree and Random Forest, the maximum tree depth is set as 2 to ensure that under-fitting issues are avoided. To achieve a smoother curve, the bagging technique is applied to the Random forest mechanism where each of the trees executes in a parallel way thus making a forest. As each tree is independent, therefore, the whole forest result is taken for the analysis (resulting in a smoother curve) To avoidover-fitting issues, we have evaluated our proposed technique using $\mathrm{K}$-fold $(\mathrm{k}=10)$ cross-validation. The first fold is evaluated with the other folds and the second time it executes, it takes the first and second fold to be compared with the rest, this goes on until $80 \%$ of training data is compared against $20 \%$ of the test data.

\section{Results and Discussion}

For experimentation, we utilize a system with Intel core 17 processor, 8 GBs of memory, and Ubuntu $12.10 \mathrm{OEM}$ as an operating system. For classification, a machine learning tool Scikitlearn (Pedregosa, et al., 2011; Black et al., 2020), is employed. To evaluate the results, standard evaluation measures i.e., precision, recall, and F-Measure are calculated to determine the accuracy of each classifier. Equations $1-4$ provide the mathematical description of accuracy,

Peer) Comput. Sci. reviewing PDF | (CS-2020:09:52627:1:1:NEW 12 Dec 2020) 
391 precision, recall, and f-measure, respectively. The terms used in Equations 1-4 are explained as 392 follows: True Positive (TP) rate shows the number of predicted positives that are correct, while 393 the False Positive (FP) rate refers to the number of predicted positives that are incorrect.

394 Similarly, True Negative (TN) rate shows the number of predicted negatives that are correct 395 while the False Negative (FN) rate refers to the number of predicted negatives that are incorrect. 396 The recall is the sensitivity for the most relevant result. F-measure is the value that estimates the 397 entire system performance by calculating the harmonic mean of precision and recall. The 398 maximum value of 1.000 for accuracy precision and recall indicates the best result (Narudin, et 399 al., 2016).

400

401

$$
\text { Accuracy }=\frac{\mathrm{TP}+\mathrm{TN}}{\mathrm{TP}+\mathrm{TN}+\mathrm{FP}+\mathrm{FN}}
$$

402

403

404

Precision denotes the proportion of Predicted Positive cases that are correctly Real Positives.

$$
\text { Precision }=\frac{\mathrm{TP}}{\mathrm{TP}+\mathrm{FP}}
$$

405

406 The recall is the proportion of Real Positive cases that are Predicted Positive

407

$$
\text { Recall }=\frac{\mathrm{TP}}{\mathrm{TP}+\mathrm{FN}}
$$

408

409

410

411

412

413

414

415

416

417

418

419

420

421

422

423

424

425

$$
\text { F-Measure }=\quad 2 * \frac{(\text { Precision } * \text { Re } \text { call })}{(\text { Pr ecision }+ \text { Re } \text { call })}
$$

Receiver Operating Characteristic (ROC) curves (Metz, 1978; Dion et al., 2020) are extensively being applied in significant researches to measure the accuracy of the machine learning models that are being trained to achieve actual performance (Bradley, 1997). Furthermore, ROC curves are applied in numerous systematic approaches that merge multiple clues, test results, etc., and are plotted and evaluated to characterize a qualitative feature of the particular. ROC is a plot wherein Y-axis is reserved for True Positive Rate (TPR) and X-axis is reserved for False Positive Rate (FPR). For all possible classifications such as the output class, the TPR rate depends on the set-up where the real classification is considered to be as positive and the number of times the classifier has predicted the result to be as positive. The FPR can be defined as how the classifier incorrectly labeled positive to those that are classified to be negative. Together the TPR and FPR values lie in-between $0-1$ ( 1 indicating an accurate prediction).

The results based on the decision tree classifier can be seen in Figure 4. The ROC curve for both classes (i.e., ransomware as class " 1 " and non-ransomware as class " 0 ") is the same having value of 0.94 which signifies the excellent prediction. However, the precision-recall curve for class 0 
426 i.e., for Non-Ransomware shows accuracy of 0.89 or $89 \%$ whereas for class 1 i.e., ransomware 427 the accuracy is 0.93. The F-measure score of the Decision Tree is 0.94 as shown in Table 4.

428

429

430

431

432

433

434

435

436

437

438

439

440

441

442

443

444

445

446

447

448

449

450

451

452

453

454

455

456

457

458

459

460

461

462

463

464

465

The results obtained using the Random Forest classifier for two classes (i.e., ransomware and non-ransomware) are shown in Figure 5 and F-measure score is illustrated in Table 5. The higher accuracy results are evident from the similar ROC curve value i.e., 0.99 for both the ransomware and non-ransomware classes.

The results depicting the performance of gradient boosting, shown in Figure 6, reveal that the ROC curve values for both the classes (i.e., ransomware and non-ransomware) as well as (i.e., 1.0) the precision-recall curves of both classes follow similar pattern of high accuracy. The Fmeasure score of the gradient boosting classifier is 0.93 for ransomware and 0.94 for nonransomware (as shown in Table 6).

The extreme gradient boosting classification model-based results are shown in Figure 7 and Table 7. The ROC curve and Precision-Recall Curve of both classes (i.e., ransomware and nonransomware) are the same (i.e., 1.0). The extreme gradient boosting based model's F-measure score is 0.97 , which is similar to the gradient boosting and random forest-based classification as shown in Table 8. The Random Forest-based classification model outperformed decision treebased classification by attaining the accuracy of 0.94 , as shown in Table 8 . However, the value of the F-measure for both the classes is 0.97 (as shown in Table 8). The model has attained an improvement of $3 \%$ than the decision tree-based classification. The model shows similar Fmeasure results of 0.97 as observed for random forest and extreme gradient boosting.

This study has demonstrated the possibility of exploiting HPCs as the potential features for ransomware detection. After analyzing the sets of ransomware and non-ransomware, the features obtained from HPCs have been analyzed to classify malicious applications into ransomware and non-ransomware categories using several machine learning algorithms such as Decision Tree, Random Forest, Gradient Boosting, and Extreme Gradient Boosting. The results of detailed experiments as stated earlier in the section have revealed that extracted hardware features play a significant role in the detection and identification of ransomware. Among all the employed machine learning classifiers, the random forest-based model and extreme gradient boosting have outperformed by yielding F-measure score of 0.97 followed by a decision tree that achieved 0.94 F-measure. Moreover, the features cache misses, task clock, and branches obtained through HPCs could be deemed as potential parameters in classifying ransomware from non-ransomware.

\section{Conclusions}

In this paper, the analysis of HPCs has been presented for Windows ransomware classification. The results have revealed that the HPCs hold the considerable potential to expose hidden indicators of the executing applications such as malicious codes and ransomware. Performance

Peer) Comput. Sci. reviewing PDF | (CS-2020:09:52627:1:1:NEW 12 Dec 2020) 
466

467

468

469

470

471

472

473

474

475

476

477

478

479

480

481

482

483

484

485

486

487

488

489

490

491

492

493

494

495

496

497

498

499

500

501

502

503

504

505

counters, i.e., cache misses, task clock, and branches have played a pivotal role in classifying ransomware in a way that if there are a high number of cache misses or a high number of branch mispredictions (where control flow becomes detectably anomalous) are good indicators that help in indicating a potential attack (Foreman, 2018). The proposed technique holds adequate potential to provide sufficient detection accuracy by attaining the F-measure score of 0.97 . This study demonstrated the possibility of exploiting HPCs as the potential feature for the detection of ransomware. However, this topic needs further investigation. In the future, we intend to scrutinize other dynamic features with the combination of call graphs to detect and classify ransomware. Moreover, the application of machine learning algorithms has shown very promising results in ransomware detection. In the future, we will expand this study to perform in-depth static analysis as well as dynamic analysis with the combination of HPCs in the detection of that ransomware that usually hides by implementing various obfuscation techniques (like packed or compressed programs, or indirect addressing (Behera \& Bhaskari, 2015)). One major challenge and limitation of this research is in ransomware detection of false positives and false negatives. Consider the case of Qwerty ransomware, which uses a benign GPG executable to perform encryption. Perhaps the proposed solution would correctly detect the GPG binary when used in this way, but we suspect it would also detect it in a benign case. Since in this work we did not evaluate benign executables, it is not clear how the system performs with software that performs encryption and/or compression tasks which is the limitation of this research that will be investigated in our future work. Moreover, the collected features are related to hardwarespecific environments, so if the system having the same architecture then the trained classification models are applicable as it is. However, in case the hardware environment is different (i.e., different architecture) then we have to retrain our machine learning models for that specific hardware environment. This is one of the limitations of our proposed work that the machine learning models trained on specific architecture are not portable across other machine architectures. Moreover, due to the modest dataset deep learning mechanism at present are not applicable. However, in the future, we intend to extend our dataset to implement more robust Auto Denoising Encoders, which comprises of multiple layers of neural networks and are bestknown for providing good accuracy.

\section{References}

Ahmadian, M. M., Shahriari, H. R. \& Ghaffarian, S. M., 2015. Connection-monitor \& connection-breaker: A novel approach for prevention and detection of high survivable ransomwares. s.1., IEEE, pp. 79-84.

Alam, M., Sinha, S., Bhattacharya, S., Dutta, S., Mukhopadhyay, D. and Chattopadhyay, A., 2020. RAPPER: Ransomware prevention via performance counters. arXiv preprint arXiv:2004.01712.

Al-rimy, B. A. S., Maarof, M. A. \& Shaid, S. Z. M., 2017. A 0-Day Aware Crypto-Ransomware Early Behavioral Detection Framework. s.1., Springer,Cham, pp. 758-766.

Peer] Comput. Sci. reviewing PDF | (CS-2020:09:52627:1:1:NEW 12 Dec 2020) 
506 Al-rimy, B.A.S., Maarof, M.A. and Shaid, S.Z.M., 2018. Ransomware threat success factors,

507

508

509

510

511

512

513

514

515

516

517

518

519

520

521

522

523

524

525

526

527

528

529

530

531

532

533

534

535

536

537

538

539

540

541

542

543

544 taxonomy, and countermeasures: A survey and research directions. Computers \& Security, 74, pp.144-166.

Alzahrani, N. and Alghazzawi, D., 2019, November. A Review on Android Ransomware Detection Using Deep Learning Techniques. In Proceedings of the 11th International Conference on Management of Digital EcoSystems (pp. 330-335)

Alzaylaee, M.K., Yerima, S.Y. and Sezer, S., 2020. DL-Droid: Deep learning based android malware detection using real devices. Computers \& Security, 89, p.101663.

Anderson, D., Thane, F. \& Alfonso, V., 1995. Next-generation intrusion detection expert system (NIDES): A summary.

Andronio, N., Zanero, S. \& Maggi, F., 2015. Heldroid: Dissecting and detecting mobile ransomware. s.1., Springer International Publishing, pp. 382-404.

Aurangzeb, S., Aleem, M., Iqbal, M. A. \& Islam, M. A., 2017. Ransomware: A Survey and Trends. Journal of Information Assurance and Security, Volume 12, pp. 048-058.

Bahador, M. B., Abadi, M. \& Tajoddin, A., 2014. Hpcmalhunter: Behavioral malware detection using hardware performance counters and singular value decomposition. " ,. s.1., IEEE, pp. 703-708.

Bahador, M.B., Abadi, M. and Tajoddin, A., 2019. HLMD: a signature-based approach to hardware-level behavioral malware detection and classification. The Journal of Supercomputing, 75(8), pp.5551-5582.

Basu, K., Krishnamurthy, P., Khorrami, F. and Karri, R., 2019. A theoretical study of hardware performance counters-based malware detection. IEEE Transactions on Information Forensics and Security, 15, pp.512-525.

Behera, C. K. \& Bhaskari, D. L., 2015. Different obfuscation techniques for code protection.. Procedia Computer Science, Volume 70, pp. 757-763.

Beneventi, F., Bartolini, A., Cavazzoni, C. \& Benini, . L., 2017. Continuous learning of HPC infrastructure models using big data analytics and in-memory processing tools. s.1., IEEE, pp. 1038-1043.

Black, P., Sohail, A., Gondal, I., Kamruzzaman, J., Vamplew, P. and Watters, P., 2020, November. API Based Discrimination of Ransomware and Benign Cryptographic Programs. In International Conference on Neural Information Processing (pp. 177-188). Springer, Cham.

Bradley, A., 1997. The use of the area under the ROC curve in the evaluation of machine learning algorithms.. Pattern Recognit, 30(7), pp. 1145-1159.

Brewer, R., 2016. Ransomware attacks: detection, prevention and cure. Network Security, 2016(9), pp. 5-9.

Chen, Q. \& Robert, A. B., 2017. Automated Behavioral Analysis of Malware A Case Study of WannaCry Ransomware.. Volume arXiv preprint arXiv:1709.08753. 
545 Chen, T., Tong, H. \& Michael, B., 2015. Xgboost: extreme gradient boosting. $R$ package, 0.4(2), 546 pp. 1-4.

547 Chen, Z., Kang, H., Yin, S. \& Kim, S., 2017. Automatic Ransomware Detection and Analysis

548 Based on Dynamic API Calls Flow Graph.. s.1., s.n., pp. 196-201.

549 Chiappetta, M., Savas, E. \& Yilmaz, C., 2016. Real time detection of cache-based side-channel $550 \quad$ attacks using hardware performance counters. Applied Soft Computing, Volume 49, pp. $551 \quad 1162-1174$.

552 Chung, M., 2019. Why employees matter in the fight against ransomware. Computer Fraud \& 553 Security, 2019(8), pp.8-11.

554 .

555

556

557

558

559

560

561

562

563

564

Das, S., Werner, J., Antonakakis, M., Polychronakis, M. and Monrose, F., 2019, May. SoK: The challenges, pitfalls, and perils of using hardware performance counters for security. In 2019 IEEE Symposium on Security and Privacy (SP) (pp. 20-38). IEEE.

Demme, J., Maycock, M., Schmitz, J., Tang, A., Waksman, A., Sethumadhavan, S. and Stolfo, S., 2013. On the feasibility of online malware detection with performance counters. ACM SIGARCH Computer Architecture News, 41(3), pp.559-570.

Davies, S.R., Macfarlane, R. and Buchanan, W.J., 2020. Evaluation of live forensic techniques in ransomware attack mitigation. Forensic Science International: Digital Investigation, 33, p.300979.

Dinakarrao, S.M.P., Amberkar, S., Bhat, S., Dhavlle, A., Sayadi, H., Sasan, A., Homayoun, H. and Rafatirad, S., 2019, June. Adversarial attack on microarchitectural events based

565

566

567 malware detectors. In Proceedings of the 56th Annual Design Automation Conference

568 2019 (pp. 1-6).

569

570

571

572

573

574

575

576

577

Dion, Y. and Brohi, S.N., 2020. An Experimental Study to Evaluate the Performance of Machine Learning Alogrithms in Ransomware Detection. J. Eng. Sci. Technol, 15(2), pp.967981.Foreman, J.C., 2018. A Survey of Cyber Security Countermeasures Using Hardware Performance Counters. arXiv preprint arXiv:1807.10868.

Friedman, J. H., 1999. Reitz Lecture. 29(2001), pp. 1189-1232.

Gazet, A., 2010. Comparative analysis of various ransomware virii. Journal in computer virology, 6(1), pp. 77-90.

Grant, L. \& Parkinson, S., 2018. Identifying File Interaction Patterns in Ransomware Behaviour. In: Guide to Vulnerability Analysis for Computer Networks and Systems. s.1.:Springer, pp. 317-335.

578 Hampton, N., Baig, Z. \& Zeadall, S., 2018. Ransomware Behavioural Analysis on Windows

579 Platform. Journal of Information Security and Applications, Volume 40, pp. 44-51.

580 Humayun, M., Jhanjhi, N.Z., Alsayat, A. and Ponnusamy, V., 2020. Internet of things and ransomware: Evolution, mitigation and prevention. Egyptian Informatics Journal. Jordan, M. I. \& Mitchell, T. M., 2015. Machine learning: Trends, perspectives, and prospects. Science, 349(6245), pp. 255-260. 
584 Jbabdi, S., Sotiropoulos, S.N., Savio, A.M., Graña, M. and Behrens, T.E., 2012. Model-based

585

586

587

588

589

590

591

592

593

594

595

596

597

598

599

600

601

602

603

604

605

606

607

608

609

610

611

612

613

614

615

616

617

618

619

620

621

622 analysis of multishell diffusion MR data for tractography: how to get over fitting problems. Magnetic resonance in medicine, 68(6), pp.1846-1855.

Kadiyala, S.P., Jadhav, P., Lam, S.K. and Srikanthan, T., 2020. Hardware Performance CounterBased Fine-Grained Malware Detection. ACM Transactions on Embedded Computing Systems (TECS), 19(5), pp.1-17

Kaur, G., Dhir, R. \& Singh, M., 2017. Anatomy of ransomware malware: detection, analysis and reporting. International Journal of Security and Networks, 12(3), pp. 188-197.

Kharraz, A., Arshad, S., Mulliner, C. \& Robertson, W. K., 2016. UNVEIL: A Large-Scale, Automated Approach to Detecting Ransomware. USENIX Security Symposium, pp. 757772.

Kharraz, A., Robertson, W., Balzarotti, D., Bilge, L. and Kirda, E., 2015, July. Cutting the gordian knot: A look under the hood of ransomware attacks. In International Conference on Detection of Intrusions and Malware, and Vulnerability Assessment (pp. 3-24). Springer, Cham

Kimberly, T., Salahuddin J, K., Aristide, F. \& Lorenzo, C., 2015. CopperDroid: Automatic Reconstruction of Android Malware Behaviors. NDSS.

Kim, D., Soh, W. \& Kim, S., 2015. Design of quantification model for prevent of cryptolocker. Indian Journal of Science and Technology, 8(19).

Kohavi, R., 1996. Scaling up the accuracy of Naive-Bayes classifiers: a decision-tree hybrid.. In $K D D, 96$ (Citeseer), p. In KDD.

Kok, S., Abdullah, A., Jhanjhi, N. and Supramaniam, M., 2019. Ransomware, threat and detection techniques: A review. Int. J. Computer Science and Network Security, 19(2), p.136.

Kuruvila, A.P., Kundu, S. and Basu, K., 2020, July. Analyzing the Efficiency of Machine Learning Classifiers in Hardware-Based Malware Detectors. In 2020 IEEE Computer Society Annual Symposium on VLSI (ISVLSI) (pp. 452-457). IEEE.

Labs, M., 2017. Threat Predictions Ransomware Infographic”, s.1.: McAfee Labs Threat Predictions report 2017.

Lee, J. K., Moon, S. Y. \& Park, J. H., 2016. CloudRPS: a cloud analysis based enhanced ransomware prevention system. The Journal of Supercomputing, 73(7), pp. 3065-3084.

Liaw, A. \& Wiener, M., 2002. Classification and regression by randomForest. $R$ news, 2(3), pp. 18-22.

Li, J., Cheng, K., Wang, S., Morstatter, F., Trevino, R.P., Tang, J. and Liu, H., 2017. Feature selection: A data perspective. ACM Computing Surveys (CSUR), 50(6), pp.1-45.

Liu, X. and Liu, J., 2014, April. A two-layered permission-based android malware detection scheme. In 2014 2nd IEEE International Conference on Mobile Cloud Computing, Services, and Engineering (pp. 142-148). IEEE. 
623

624

625

626

627

628

629

630

631

632

633

634

635

636

637

638

639

640

641

642

643

644

645

646

647

648

649

650

651

652

653

654

655

656

657

658

659

660

Maiorca, D., Mercaldo, F., Giacinto, G., Visaggio, C.A. and Martinelli, F., 2017, April. RPackDroid: API package-based characterization and detection of mobile ransomware. In Proceedings of the symposium on applied computing (pp. 1718-1723).

Malone, C., Zahran, M. and Karri, R., 2011, October. Are hardware performance counters a cost effective way for integrity checking of programs. In Proceedings of the sixth ACM workshop on Scalable trusted computing (pp. 71-76).

Martinelli, F., Mercaldo, F. \& Saracino, A., 2017. Bridemaid: An hybrid tool for accurate detection of android malware. .. s.1., In Proceedings of the 2017 ACM on Asia Conference on Computer and Communications Security ACM, pp. 899-901.

Maurya, A., Kumar, N., Agrawal, A. \& Khan, R., 2018. Ransomware: Evolution, Target and Safety Measures. International Journal of Computer Sciences and Engineering, 6(1). McLaughlin, N., Martinez del Rincon, J., Kang, B., Yerima, S., Miller, P., Sezer, S., Safaei, Y., Trickel, E., Zhao, Z., Doupé, A. and Joon Ahn, G., 2017, March. Deep android malware detection. In Proceedings of the Seventh ACM on Conference on Data and Application Security and Privacy (pp. 301-308).

Metz, C. E., 1978. Basic principles of ROC analysis. Seminars in nuclear medicine, 8(4).

Micro, T., 2016. Ransomware, s.1.: Trend Micro Incorporated Labs report, 2016.

Mucci, P. J., Browne, S., Deane, C. \& Ho, G., 1999. PAPI: A portable interface to hardware performance counters. s.1., Proceedings of the department of defense HPCMP users group conference.

Narudin, F. A., Feizollah, A., Anuar, N. B. \& Gani, A., 2016. Evaluation of machine learning classifiers for mobile malware detection. Soft Computing, 20(1), pp. 343-357.

Or-Meir, O., Nissim, N., Elovici, Y. and Rokach, L., 2019. Dynamic malware analysis in the modern era-A state of the art survey. ACM Computing Surveys (CSUR), 52(5), pp.1-48.

Pedregosa, F., Varoquaux, G., Gramfort, A., Michel, V., Thirion, B., Grisel, O., Blondel, M., Prettenhofer, P., Weiss, R., Dubourg, V. and Vanderplas, J., 2011. Scikit-learn: Machine learning in Python. the Journal of machine Learning research, 12, pp.2825-2830.

Perf, L., 2016. Linux profiling with performance counters.

Ramesh, G. and Menen, A., 2020. Automated dynamic approach for detecting ransomware using finite-state machine. Decision Support Systems, 138, p.113400.

Scalas, M., Maiorca, D., Mercaldo, F., Visaggio, C.A., Martinelli, F. and Giacinto, G., 2019. On the effectiveness of system API-related information for Android ransomware detection. Computers \& Security, 86, pp.168-182.

Sgandurra, D., Muñoz-González, . L., Mohsen, . R. \& Lupu, E. C., 2016. Automated Dynamic Analysis of Ransomware: Benefits, Limitations and use for Detection. arXiv preprint arXiv:1609.03020.

Simonyan, K. and Zisserman, A., 2014. Very deep convolutional networks for large-scale image recognition. arXiv preprint arXiv:1409.1556.

Peer] Comput. Sci. reviewing PDF | (CS-2020:09:52627:1:1:NEW 12 Dec 2020) 
661

662

663

664

665

666

667

668

669

670

671

672

673

674

675

676

677

678

679

680

681

682

683

684

685

686

687

688

689

690

691

692

693

694

695

696

Singh, B., Evtyushkin, D., Elwell, J., Riley, R. and Cervesato, I., 2017, April. On the detection of kernel-level rootkits using hardware performance counters. In Proceedings of the 2017 ACM on Asia Conference on Computer and Communications Security (pp. 483-493).

Song, S., Kim, B. \& Lee, S., 2016. The effective ransomware prevention technique using process monitoring on android platform.

Tan, P.-N., Steinbach, . M. \& Kumar, V., 2006. Classification: basic concepts, decision trees, and model evaluation.. Introduction to data mining, Volume 1, pp. 145-205.

Wang, X., Chai, S., Isnardi, M., Lim, S. and Karri, R., 2016. Hardware performance counterbased malware identification and detection with adaptive compressive sensing. ACM Transactions on Architecture and Code Optimization (TACO), 13(1), pp.1-23

Weaver, V. M., 2013. Linux perf_event features and overhead.. The 2nd International Workshop on Performance Analysis of Workload Optimized Systems, FastPath., Volume 13.

Victoriano, O.B., 2019, October. Exposing Android Ransomware using Machine Learning. In Proceedings of the 2019 International Conference on Information System and System Management (pp. 32-37).

Xu, Z., Ray, S., Subramanyan, P. \& Malik, S., 2017. Malware detection using machine learning based analysis of virtual memory access patterns.. In Proceedings of the Conference on Design, Automation \& Test in Europe European Design, pp. 169-174.

Xuan, S., Liu, G., Li, Z., Zheng, L., Wang, S. and Jiang, C., 2018, March. Random forest for credit card fraud detection. In 2018 IEEE 15th International Conference on Networking, Sensing and Control (ICNSC) (pp. 1-6). IEEE.

Yang, . T., Yang, Y., Qian, K. \& Tao, L., 2015. Automated detection and analysis for android ransomware. s.1., IEEE, pp. 1338-1343.

Zavarsky, P. \& Lindskog, D., 2016. Experimental Analysis of Ransomware on Windows and Android Platforms: Evolution and Characterization. Procedia Computer Science, 94(2016), pp. 465-472.

Zhang, M., Xu, B. \& Wang, D., 2015. An anomaly detection model for network intrusions using one-class SVM and scaling strategy. Cham, Springer, pp. 267-278.

Zhang, H., Xiao, X., Mercaldo, F., Ni, S., Martinelli, F. and Sangaiah, A.K., 2019. Classification of ransomware families with machine learning based on $\mathrm{N}$-gram of opcodes. Future Generation Computer Systems, 90, pp.211-221.

Zhang, X., Zhao, J. and LeCun, Y., 2015. Character-level convolutional networks for text classification. In Advances in neural information processing systems (pp. 649-657).

Zhou, B., Gupta, A., Jahanshahi, R., Egele, M. and Joshi, A., 2018, May. Hardware performance counters can detect malware: Myth or fact?. In Proceedings of the 2018 on Asia Conference on Computer and Communications Security (pp. 457-468).

PeerJ Comput. Sci. reviewing PDF | (CS-2020:09:52627:1:1:NEW 12 Dec 2020) 


\section{Table $\mathbf{1}$ (on next page)}

Comprehensive Comparison of the state-of-the-art approaches along with their key points, drawbacks and implementation design approach 


\begin{tabular}{|c|c|c|c|}
\hline Reference & Methodology & Strengths & Limitations \\
\hline $\begin{array}{l}\text { Demme et al., } \\
\text { (2013) }\end{array}$ & $\begin{array}{l}\text { - Dynamic approach } \\
\text { - Android Malware } \\
\text { detection with } \\
\text { performance counters } \\
\text { Applied ML algorithms } \\
\text { (KNN, Decision tree) }\end{array}$ & $\begin{array}{l}\text { - Major support is that runtime } \\
\text { behavior can capture using HW } \\
\text { performance counters are } \\
\text { essential to detect malware } \\
90 \% \text { accuracy with } 3 \% \text { FP }\end{array}$ & $\begin{array}{l}\text { - Able to detect some } \\
\text { variants whereas some } \\
\text { were not detected } \\
\text { - Malware label data } \\
\text { might not accurate }\end{array}$ \\
\hline $\begin{array}{l}\text { Kharraz et al., } \\
(2015)\end{array}$ & $\begin{array}{l}\text { - Analyzed } 15 \text { ransomware } \\
\text { families } \\
\text { - Proposed various } \\
\text { mitigation approaches to } \\
\text { decoy resources to detect } \\
\text { malicious file access. }\end{array}$ & $\begin{array}{l}\text { - Provide evolution-based study } \\
\text { of RW attacks from a long-term } \\
\text { study 2006-2014 } \\
\text { - Detailed analysis of Bitcoin for } \\
\text { monetization }\end{array}$ & $\begin{array}{l}\text { - Assumed that every file } \\
\text { system access to delete } \\
\text { or encrypt decoy } \\
\text { resources } \\
\text { - However, they didn't } \\
\text { implement any concrete } \\
\text { solution to detect or } \\
\text { defend against these } \\
\text { attacks }\end{array}$ \\
\hline $\begin{array}{l}\text { Kim and Kim } \\
(2015)\end{array}$ & $\begin{array}{l}\text { present a quantification } \\
\text { model based on social } \\
\text { engineering technique to } \\
\text { avoid and identify any } \\
\text { cryptographic operations } \\
\text { in the local drive }\end{array}$ & $\begin{array}{l}\text { explains the file-based intrusion } \\
\text { detection system and IP } \\
\text { traceback algorithm }\end{array}$ & $\begin{array}{l}\text { - Lack of experimental } \\
\text { results } \\
\text { - Suggests guidelines } \\
\text { online }\end{array}$ \\
\hline $\begin{array}{l}\text { Narudin et al., } \\
(2016)\end{array}$ & $\begin{array}{l}\text { - Machine learning-based } \\
\text { study } \\
\text { - Filter TCP packets, extract } \\
\text { network traffic features } \\
\text { - Evaluate Bayes, Random } \\
\text { Forest, KNN, J48, \& MLP }\end{array}$ & $\begin{array}{l}\text { - Accurate detection based on } \\
\text { ML classifiers. } \\
\text { - BN and RF produces } 99.97 \% \\
\text { TPR } \\
\text { - Bayes, MLP with ROC } 0.995 \\
\text { and RF with } 0.991\end{array}$ & $\begin{array}{l}\text { - Applicable for Android } \\
\text { platform only }\end{array}$ \\
\hline $\begin{array}{l}\text { Zavarsky and } \\
\text { Lindskog } \\
(2016)\end{array}$ & $\begin{array}{l}\text { - the life cycle of Windows- } \\
\text { based Ransomware study. } \\
\text { - Implement basic static and } \\
\text { basic dynamic } \\
\text { - MD5 method, Cuckoo } \\
\text { Sandbox used. } \\
\text { - For android Analyze } \\
\text { AndroidManifest.xml, } \\
\text { administrative privilege } \\
\text { - For Windows analyze } \\
\text { Filesystems, registry } \\
\text { activities, and network } \\
\text { operations }\end{array}$ & $\begin{array}{l}\text { - Explained the detailed analysis, } \\
\text { working, and functionality of } \\
\text { Ransomware } \\
\text { - Performed analysis on both the } \\
\text { Windows and Android-based } \\
\text { RW } \\
\text { - PEiD tool is used for windows } \\
\text { ransomware detection }\end{array}$ & $\begin{array}{l}\text { - Performed only basic } \\
\text { static and dynamic } \\
\text { analysis. } \\
\text { - No machine learning- } \\
\text { based approach to detect } \\
\text { zero-day ransomware } \\
\text { - Lack of experimental } \\
\text { analysis }\end{array}$ \\
\hline $\begin{array}{l}\text { Song, et al., } \\
(2016)\end{array}$ & $\begin{array}{l}\text { Proposed techniques on } \\
\text { three modules: } \\
\text { Configuration, Monitors, } \\
\text { and Processes sing } \\
\text { - the hash information } \\
\text { method is used for } \\
\text { detection of CryptoLocker } \\
\text { type ransomware }\end{array}$ & $\begin{array}{l}\text { - The proposed technique } \\
\text { monitors the processes and } \\
\text { specific file directories } \\
\text { monitor file events using } \\
\text { statistical methods on Processor } \\
\text { usage, Memory usage, and I/O } \\
\text { rates }\end{array}$ & $\begin{array}{l}\text { - Not applicable for } \\
\text { Windows-based } \\
\text { ransomware } \\
\text { - No classifier is used } \\
\text { - Does not install } \\
\text { applications and execute } \\
\text { for prevention and } \\
\text { detection } \\
\text { - Results are not analyzed } \\
\text { quantitatively }\end{array}$ \\
\hline
\end{tabular}




\begin{tabular}{|c|c|c|c|}
\hline $\begin{array}{l}\text { Kharraz et al., } \\
(2016)\end{array}$ & $\begin{array}{l}\text { - dynamic approach } \\
\text { - Monitors file system I/O } \\
\text { activity } \\
\text { - Detect screen locking } \\
\text { mechanism, } \\
\text { - used Tesseract-OCR }\end{array}$ & $\begin{array}{l}\text { - new ransomware family were } \\
\text { detected that was not detected } \\
\text { previously } \\
\text { - The long-term study analyzed } \\
148223 \text { malware samples and } \\
\text { correctly detect and verified } \\
13637 \text { ransomware samples } \\
\text { - } 96.3 \% \text { TP rate and } 0 \text { FPs }\end{array}$ & $\begin{array}{l}\text { Accuracy is not that } \\
\text { good. For example, the } \\
\text { system correctly detects } \\
7,572 \text { ransomware } \\
\text { whereas only one } \\
\text { unknown was detected }\end{array}$ \\
\hline $\begin{array}{l}\text { Sgandurra et } \\
\text { al., (2016) }\end{array}$ & $\begin{array}{l}\text { - Dynamically monitor file } \\
\text { system activity on } \\
\text { windows platform } \\
\text { - Classify between } \\
\text { goodware and } \\
\text { ransomware using ML } \\
\text { - Mutual Information and } \\
\text { Regularized Logistic } \\
\text { Regression classifier used. } \\
\text { - Proposed machine } \\
\text { learning approach } \\
\text { EldeRan }\end{array}$ & $\begin{array}{l}\text { - } \mathrm{e} \square \text { ective and entirely } \\
\text { automated tool to analyze new } \\
\text { software and enhance the } \\
\text { detection capabilities of AV } \\
\text { software } \\
\text { - registry key and API calls are } \\
\text { the two classes with the most } \\
\text { relevant features. } \\
\text { - EldeRan achieves ROC curve } \\
\text { of } 0.995 \text {, detection rate } 96.3 \%\end{array}$ & $\begin{array}{l}\text { - Despite good results, } \\
\text { EldeRan still not be used } \\
\text { as a replacement for AV } \\
\text { - the current settings have } \\
\text { no other applications } \\
\text { running in the VM, } \\
\text { except the ones coming } \\
\text { with a fresh installation } \\
\text { of Windows, } \\
\text { - initial dataset was larger } \\
\text { - Unable to analyze RW } \\
\text { that shows silent } \\
\text { behavior, or wait for the } \\
\text { user to do something }\end{array}$ \\
\hline $\begin{array}{l}\text { Chen and } \\
\text { Robert, (2017) }\end{array}$ & $\begin{array}{l}\text { - Dynamic behavioral } \\
\text { analysis of wanna cry } \\
\text { - Present a method to } \\
\text { extract features of } \\
\text { malware from hosts logs } \\
\text { - TF-IDF approach gives } \\
\text { better results for analyzing } \\
\text { wanna cry }\end{array}$ & $\begin{array}{l}\text { - Research helps in further } \\
\text { manual analysis of logs from } \\
\text { ambient system logs in forensic } \\
\text { efforts. } \\
\text { - Automatically generate } \\
\text { behavior analysis of malware } \\
\text { samples from sandbox log data }\end{array}$ & $\begin{array}{l}\text { - Presentation and } \\
\text { experimented results are } \\
\text { outside the scope of the } \\
\text { paper } \\
\text { - Study not help in } \\
\text { analyzing automatic } \\
\text { pattern generation }\end{array}$ \\
\hline $\begin{array}{l}\text { Al-rimy et al., } \\
(2017)\end{array}$ & $\begin{array}{l}\text { - Machine learning n-gram, } \\
\text { EFCM, } \\
\text { - Information Gain, } \\
\text { - Sliding window } \\
\text { - Static + dynamic conf } \\
\text { - SVM for behavioral } \\
\text { detection }\end{array}$ & $\begin{array}{l}\text { Proposed framework inclines to } \\
\text { share the pre-encryption data } \\
\text { space as the main defense step } \\
\text { against crypto-ransomware } \\
\text { attacks }\end{array}$ & $\begin{array}{l}\text { - No classification } \\
\text { - No experimental work } \\
\text { - No results evaluation } \\
\text { details }\end{array}$ \\
\hline $\begin{array}{l}\text { Bahador et al., } \\
(2019)\end{array}$ & $\begin{array}{l}\text { Presents a two-stage } \\
\text { heuristic matching } \\
\text { strategy signature-based } \\
\text { approach to hardware- } \\
\text { level behavioral malware } \\
\text { detection and } \\
\text { classification }\end{array}$ & $\begin{array}{l}\text { - HLMD approach can detect } \\
\text { malicious applications at the } \\
\text { beginning of the execution and } \\
\text { can achieve an average } \\
\text { precision, recall, and F-measure } \\
\text { of } 95.19 \%, 89.96 \% \text {, and } \\
92.50 \% \text {, respectively }\end{array}$ & $\begin{array}{l}\text { - Their approach is } \\
\text { suitable for independent } \\
\text { malicious programs } \\
\text { (worms, Trojans and } \\
\text { bots) that can be run } \\
\text { standalone without } \\
\text { having to be attached to } \\
\text { a host program } \\
\text { - Not applicable for } \\
\text { Ransomware }\end{array}$ \\
\hline $\begin{array}{l}\text { Dion et al., } \\
(2020)\end{array}$ & $\begin{array}{l}\text { - analyzed the opcodes and } \\
\text { measures their } \\
\text { frequencies. } \\
\text { - Compare the performance } \\
\text { of supervised machine } \\
\text { learning algorithms for } \\
\text { ransomware classification }\end{array}$ & $\begin{array}{l}\text { - Experimental analysis of } \\
\text { Random Forest, Gradient } \\
\text { Boosting Decision Tree } \\
\text { (GBDT), Neural Network using } \\
\text { Multilayer Perceptron and three } \\
\text { types of Support Vector } \\
\text { Machine (SVM) were }\end{array}$ & $\begin{array}{l}\text { - Authors mentioned that } \\
\text { the experimental } \\
\text { platform can be able to } \\
\text { identify only exe or ddl } \\
\text { format ransomware } \\
\text { - Only supervised machine } \\
\text { learning applied }\end{array}$ \\
\hline
\end{tabular}




\begin{tabular}{|c|c|c|c|}
\hline & & $\begin{array}{l}\text { performed } \\
\text { - Random Forest and GBDT } \\
\text { outperformed }\end{array}$ & \\
\hline $\begin{array}{l}\text { Kadiyala et } \\
\text { al., } \\
(2020)\end{array}$ & $\begin{array}{l}\text { - Malware Analysis using } \\
\text { Hardware Performance } \\
\text { Counters } \\
\text { - Proposed a three-step } \\
\text { methodology included i) } \\
\text { extracting the HPCs ii) } \\
\text { finding maximum } \\
\text { variance through reducing } \\
\text { fine-grained data iii) apply } \\
\text { ML algorithms }\end{array}$ & $\begin{array}{l}\text { - extract the HPCs for each } \\
\text { system call during the runtime } \\
\text { of the program using perf } \\
\text { libraries along with CoreSight } \\
\text { Access Libraries that allows to } \\
\text { interact directly through APIs } \\
\text { - detection rate } 98.4 \%\end{array}$ & $\begin{array}{l}\text { - } \text { suitable for linux } \\
\text { environment } \\
\text { - Training set is small } \\
\text { - Monitored only four } \\
\text { hardware performance } \\
\text { counters } \\
\text { - } 3.1 \% \text { false positive }\end{array}$ \\
\hline $\begin{array}{l}\text { Alam et al. } \\
2020\end{array}$ & $\begin{array}{l}\text { - Dynamic Analysis } \\
\text { - Implement Artificial } \\
\text { Neural Network and Fast } \\
\text { Fourier Transformation } \\
\text { - Disk encryption detection } \\
\text { module process used }\end{array}$ & $\begin{array}{l}\text { - Two-step detection framework } \\
\text { named as RAPPER } \\
\text { - an accurate, fast, and reliable } \\
\text { solution to detect ransomware. } \\
\text { - Used minimal tracepoints } \\
\text { - Provide a comprehensive } \\
\text { solution to tackle standard } \\
\text { benchmark, } \\
\text { - disk encryption and regular high } \\
\text { - computational processes } \\
\text { - HPCs were used to analyze files } \\
\text { using perf tool }\end{array}$ & $\begin{array}{l}\text { - Observe } 5 \text { events of } \\
\text { HPCs only i.e., instruction, } \\
\text { cache-references, cache- } \\
\text { misses, branches, and } \\
\text { branch-misses } \\
\text { - Analyze and present all } \\
\text { the case studies by giving a } \\
\text { comparison with } \\
\text { WannaCry only } \\
\text { - Lack of detailed } \\
\text { experimental results and } \\
\text { accuracies. }\end{array}$ \\
\hline Our Approach & $\begin{array}{l}\text { - Dynamic Analysis of } \\
\text { Hardware Performance } \\
\text { counters } \\
\text { - Performed classification } \\
\text { techniques on Windows- } \\
\text { based executable files }\end{array}$ & $\begin{array}{l}\text { - Apply ML algorithm such as } \\
\text { RF, Decision Tree, Gradient } \\
\text { Boosting, Extreme Gradient } \\
\text { Boosting } \\
\text { - Attained F-measure score of } \\
0.97 \\
\text { - Random Forest and Extreme } \\
\text { Gradient boosting outperformed }\end{array}$ & $\begin{array}{l}\text { - Dataset was initially large } \\
\text { but after preprocessing } \\
\text { remain small dataset } \\
\text { - Only supervised machine } \\
\text { learning techniques applied }\end{array}$ \\
\hline
\end{tabular}

1 


\section{Table 2 (on next page)}

Features Set used in this work for performance evaluation (HPCs) 


\begin{tabular}{|c|c|c|}
\hline S.no & Hardware Features & Description \\
\hline 1 & Task-clock & $\begin{array}{l}\text { The task-clock shows the amount of time spent on the task (Kuznetsova et } \\
\text { al. 2017) }\end{array}$ \\
\hline 2 & CPU utilization & CPU-clock is based on the total time spent on the CPU. \\
\hline 3 & Context Switching & $\begin{array}{l}\text { explains how many times the software switched off the CPU from one } \\
\text { process/thread to another (Kuznetsova et al. 2017) }\end{array}$ \\
\hline 4 & CPU Migration & $\begin{array}{l}\text { CPU migration describes equality in a workload distribution across all } \\
\text { cores. (Kuznetsova et al. 2017) }\end{array}$ \\
\hline 5 & Page Faults & $\begin{array}{l}\text { Page-faults occur when a program's virtual content has to be copied to the } \\
\text { physical memory (Kuznetsova et al. 2017) }\end{array}$ \\
\hline 6 & Instructions per cycle & The average number of instructions executed for each clock cycle \\
\hline 7 & Branch & $\begin{array}{l}\text { A branch is an instruction in a computer program that can cause a } \\
\text { computer to begin executing a different instruction sequence and thus } \\
\text { deviate from its default behavior of executing instructions in order }\end{array}$ \\
\hline 8 & Branch Misses & $\begin{array}{l}\text { Branch misprediction occurs when a processor mispredicts the next } \\
\text { instruction to process in branch prediction, which is aimed at speeding up } \\
\text { execution. }\end{array}$ \\
\hline 9 & Cycles & $\begin{array}{l}\text { Perf-CPU-cycles is a count of CPU cycles that traces to a hardware } \\
\text { counter (Flater \& Flater, 2014) }\end{array}$ \\
\hline 10 & Cache Misses & $\begin{array}{l}\text { Cache misses is a state of not getting data that is being processed by a } \\
\text { component or application that is not found in the cache. }\end{array}$ \\
\hline 11 & Total Time elapsed & It's the total execution time in seconds \\
\hline
\end{tabular}




\section{Table 3 (on next page)}

Features Rank List 


\begin{tabular}{|c|c|l|}
\hline Rank & Score & \multicolumn{1}{|c|}{ Feature } \\
\hline 1 & 0.20145 & cache misses \\
\hline 2 & 0.181887 & taskClock \\
\hline 3 & 0.153562 & Branches \\
\hline 4 & 0.10867 & secondsTimeElapsed \\
\hline 5 & 0.086973 & Instructions \\
\hline 6 & 0.085666 & branchMisses \\
\hline 7 & 0.044272 & contextSwitches \\
\hline 8 & 0.042727 & pageFaults \\
\hline 9 & 0.040087 & CPU migration \\
\hline 10 & 0.028564 & Cycles \\
\hline 11 & 0.026142 & CPUsUtilized \\
\hline
\end{tabular}




\section{Table 4 (on next page)}

Decision Tree precision, recall and F-measure score for malware classes $(0,1)$ 
1

\begin{tabular}{|llll|}
\hline Malware Class & Precision & Recall & F- Measure \\
\hline Ransomware (class label 1) & 1.0 & 0.88 & 0.93 \\
\hline Non- Ransomware (class label 0) & 0.89 & 1.0 & 0.94 \\
\hline
\end{tabular}

2

3 


\section{Table 5 (on next page)}

Random Forest Precision Recall and F-Measure Score against classes 0 and 1 
1

\begin{tabular}{|llcl|}
\hline Malware Class & Precision & Recall & F- Measure \\
\hline Ransomware (class label 1) & 1.0 & 0.94 & 0.97 \\
\hline Non- Ransomware (class label 0) & 0.94 & 1.0 & 0.97 \\
\hline
\end{tabular}

2 


\section{Table 6(on next page)}

Gradient Boosting precision, recall and F-measure score for malware classes 
1

\begin{tabular}{|llcl|}
\hline Malware Class & Precision & Recall & F- Measure \\
\hline Ransomware (class label 1) & 1.0 & 0.88 & 0.93 \\
\hline Non- Ransomware (class label 0) & 0.89 & 1.0 & 0.94 \\
\hline
\end{tabular}

2

3 


\section{Table 7 (on next page)}

Extreme Gradient Boosting precision, recall and F-measure score for malware 
1

\begin{tabular}{|llcc|}
\hline Malware Class & Precision & Recall & F- Measure \\
\hline Ransomware (class label 1) & 1.0 & 0.94 & 0.97 \\
\hline Non- Ransomware (class label 0) & 0.94 & 1.0 & 0.97 \\
\hline
\end{tabular}

2

3 


\section{Table 8(on next page)}

Four classifiers result and their comparison F-measure score 


\begin{tabular}{|ll|}
\hline Classifier & F- Measure \\
\hline Decision Tree & 0.94 \\
\hline Random Forest & 0.97 \\
\hline Gradient Boosting & 0.94 \\
\hline Extreme Gradient Boosting & 0.97 \\
\hline
\end{tabular}

2

3

4 


\section{Figure 1}

Estimation and projection of losses (in Billions USD) caused by different ransomware between 2015-2021 (Ramesh and Menen, 2020; Chung, 2019)

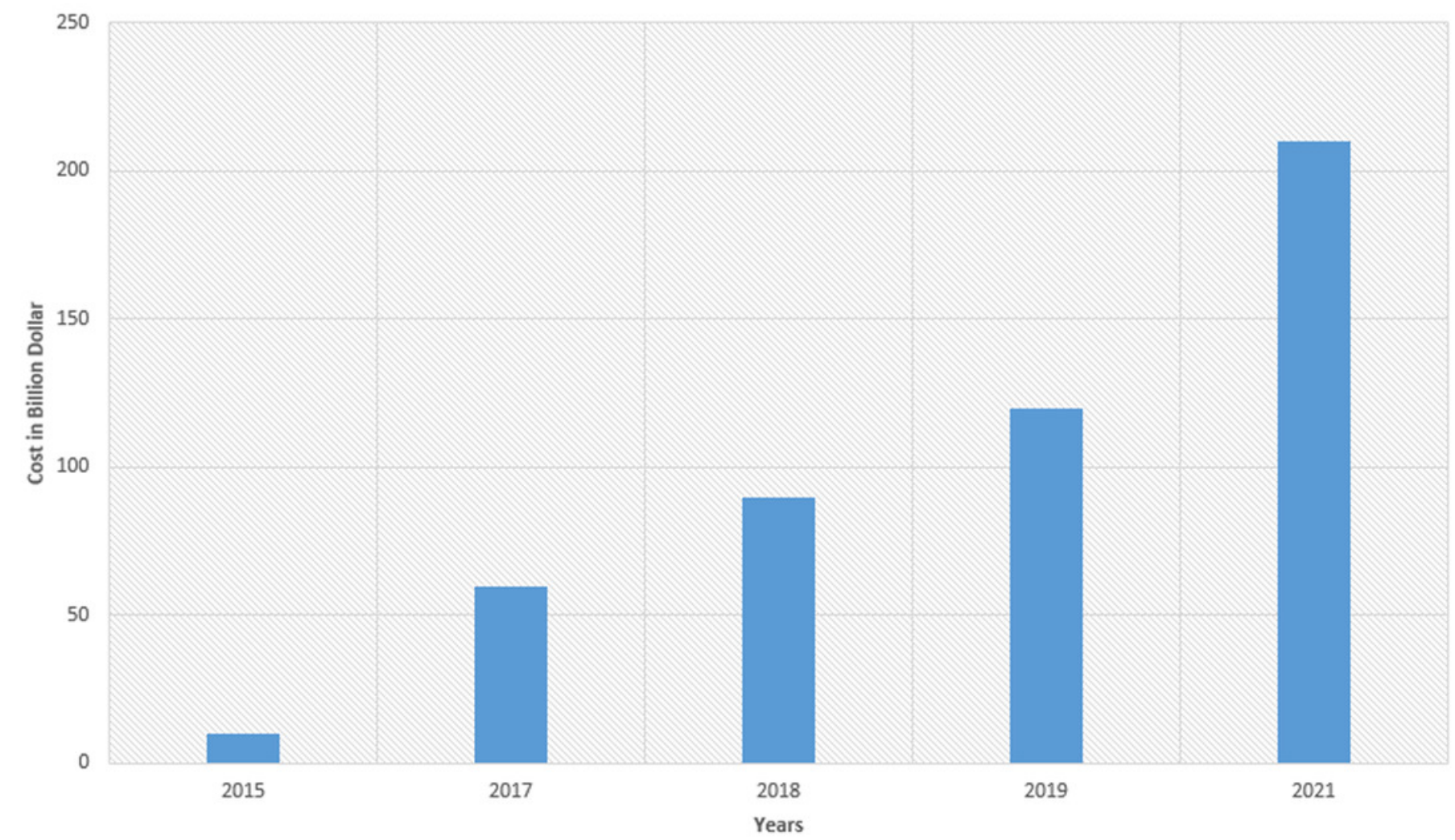


Figure 2

Feature Set correlation analysis

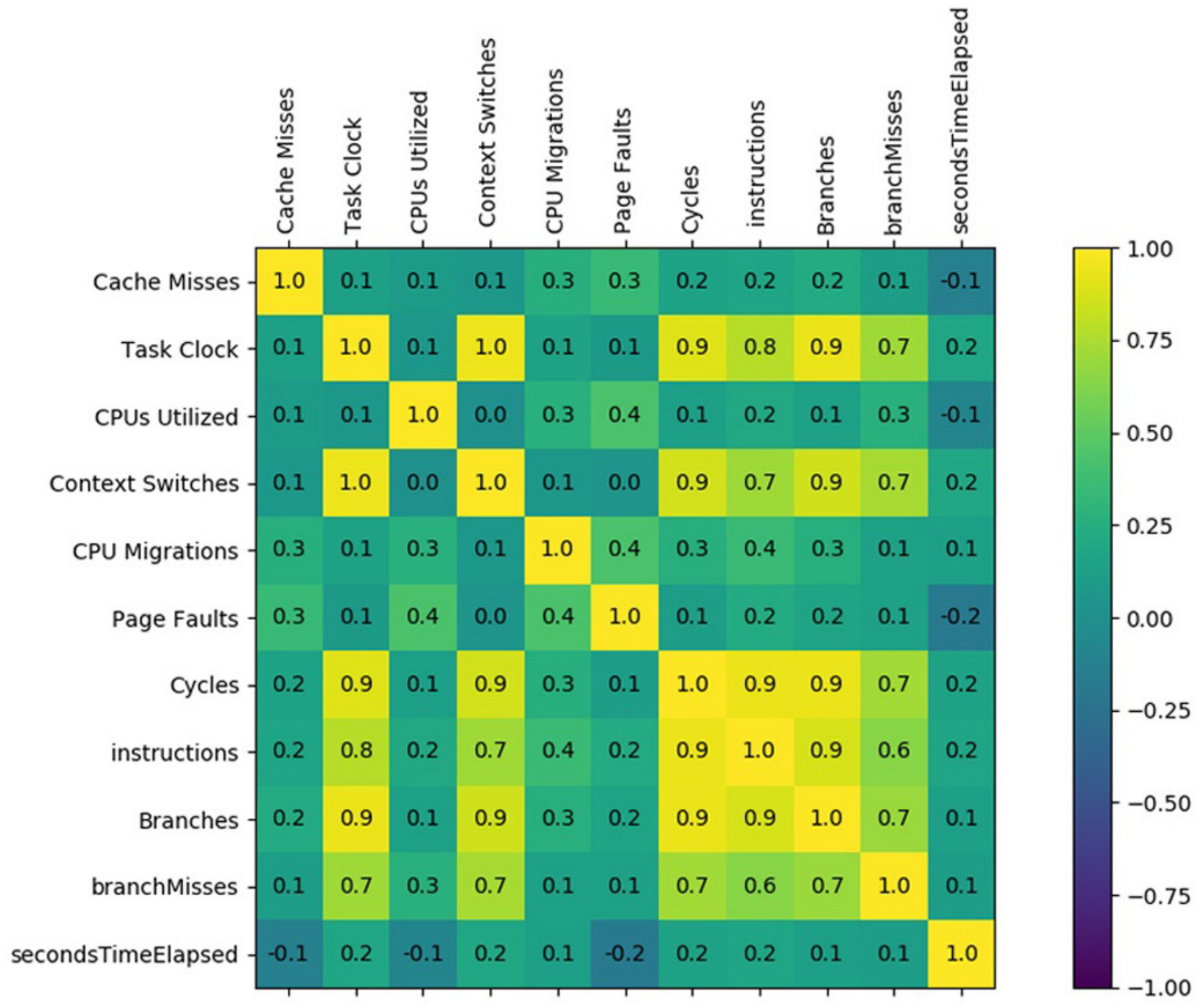


Figure 3

Workflow of training and testing phases

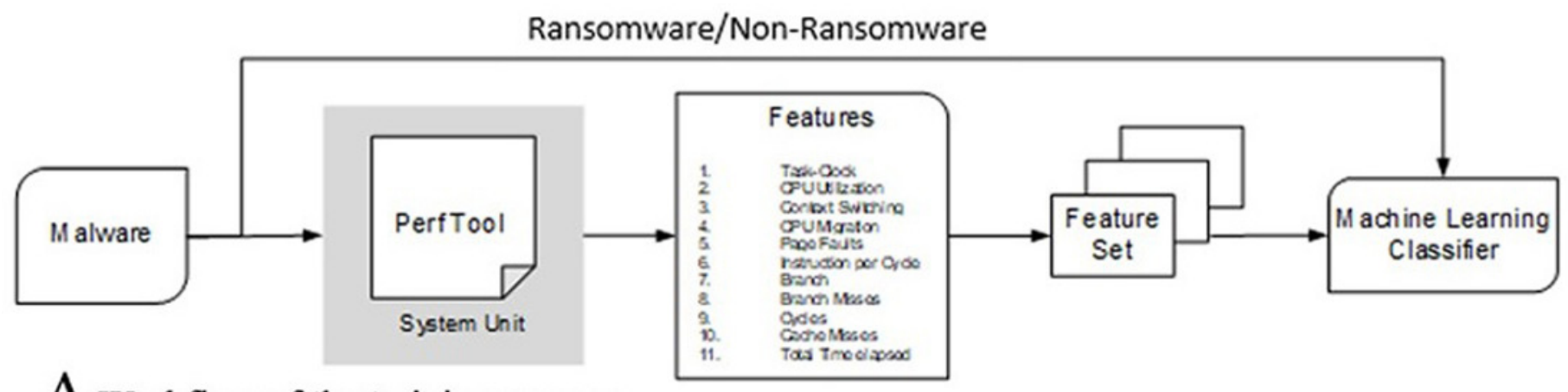

A Workflow of the training process

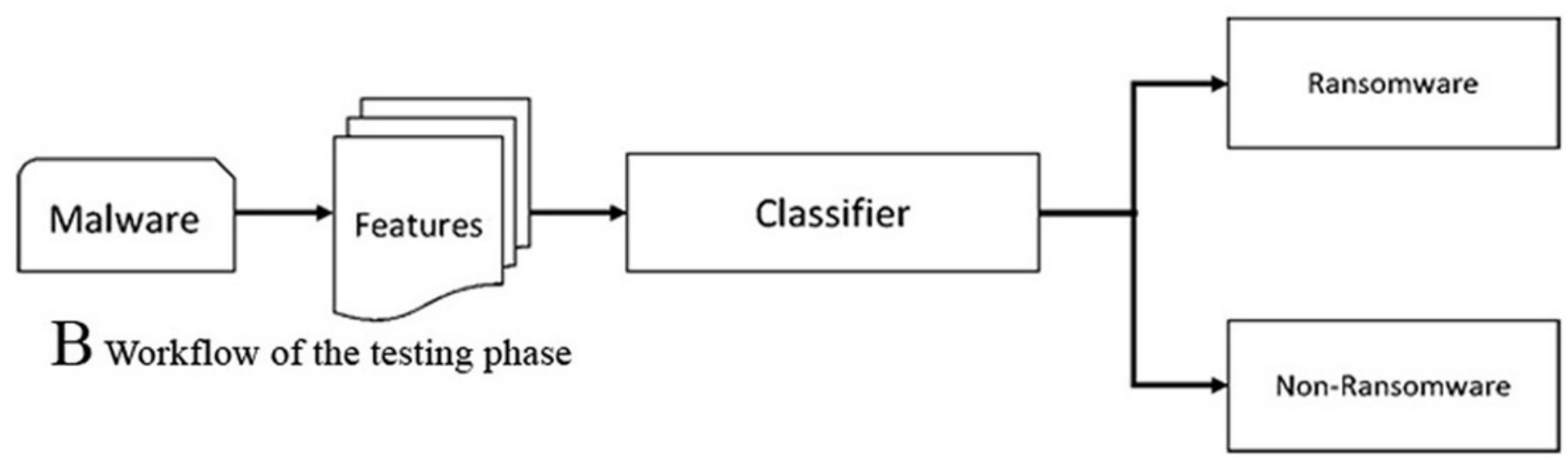


Figure 4

\section{Decision Tree performance metrics}
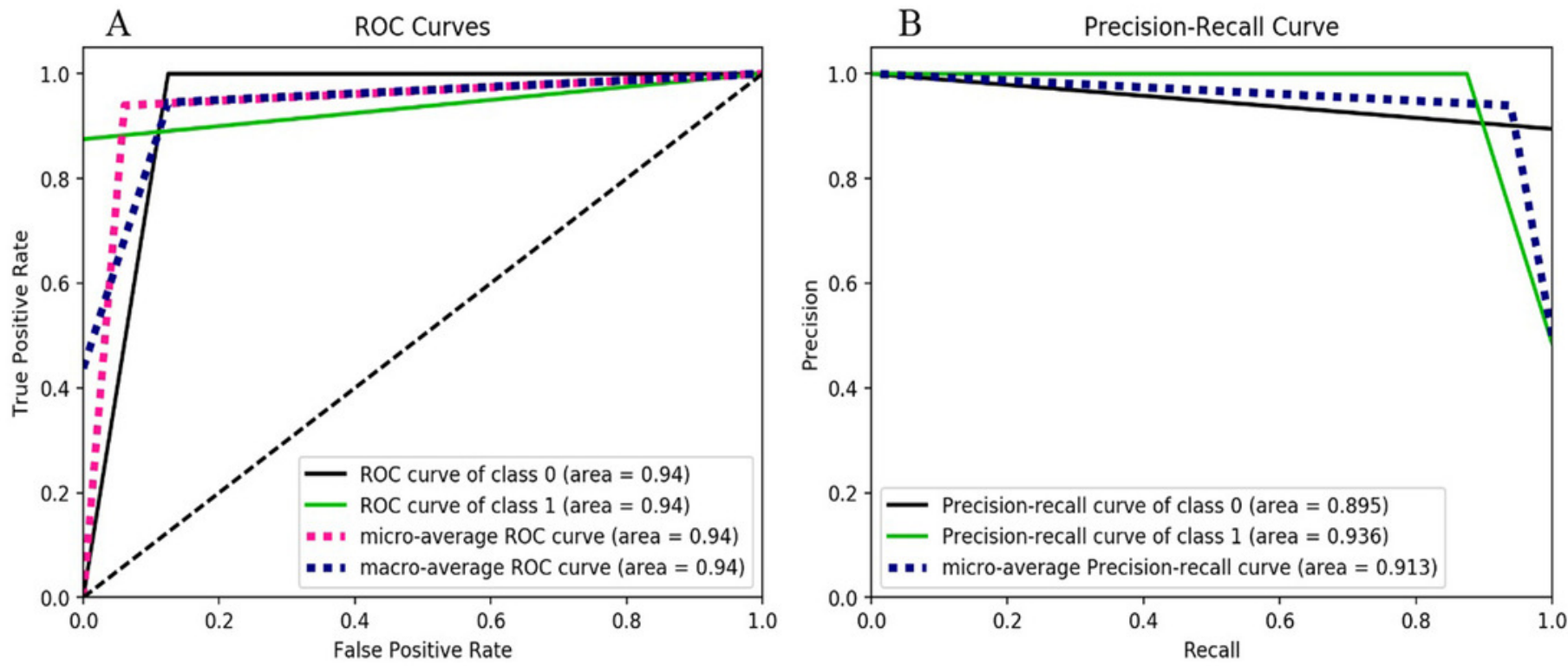
Figure 5

\section{Random Forest performance metrics}
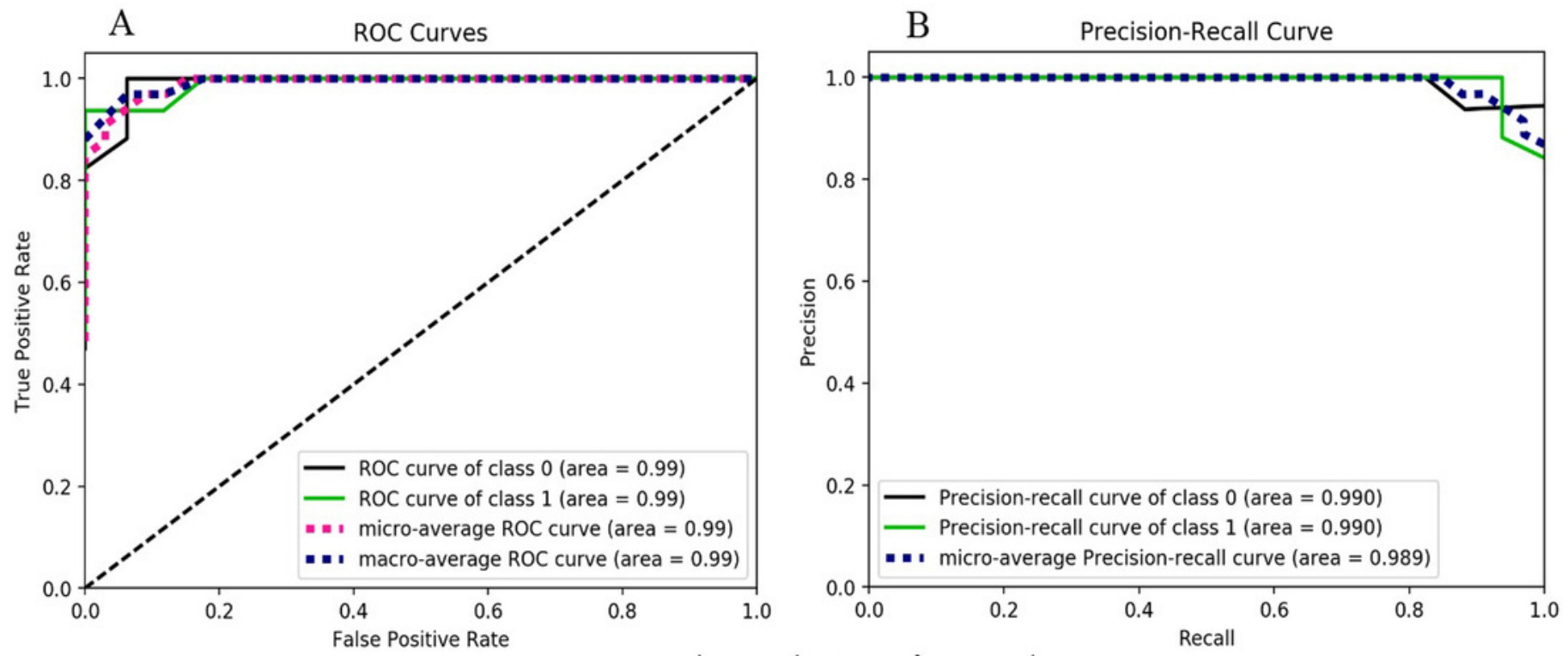
Figure 6

\section{Gradient Boosting performance metrics}
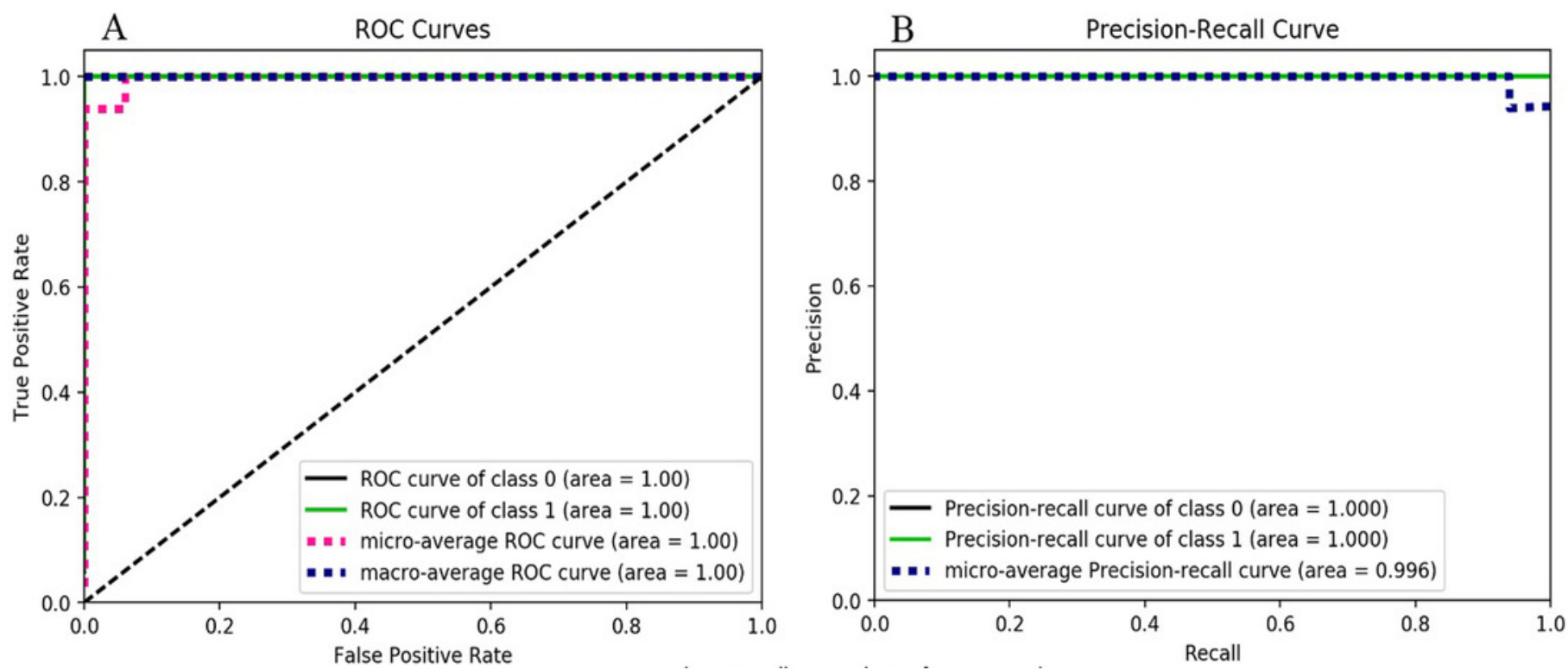
Figure 7

\section{Extreme Gradient Boosting performance metrics}
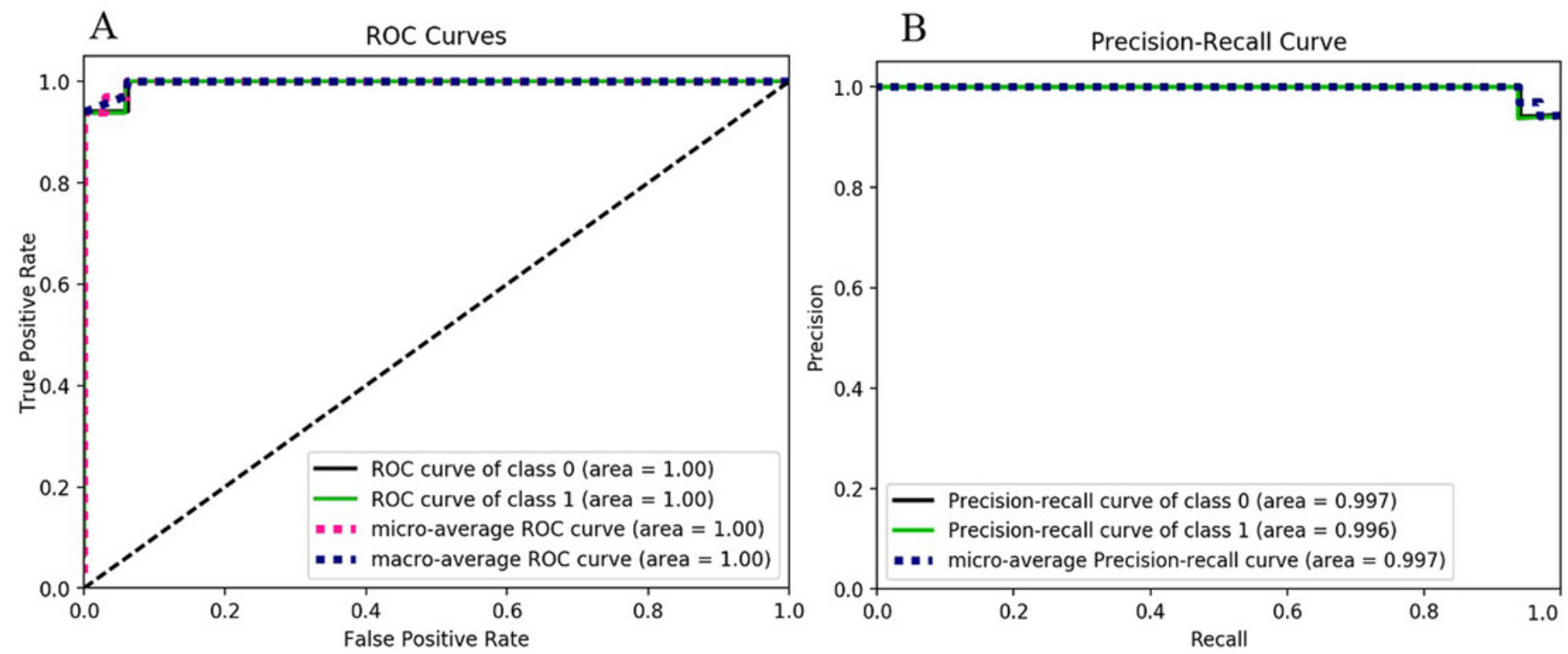